\title{
Anomalous gauge couplings in $W / Z$ exclusive pair production at the LHC
}

\section{E. Chapon*}

CEA Saclay / Irfu / SPP

E-mail: emilien.chaponecern.ch

\section{Royon}

CEA Saclay / Irfu / SPP

E-mail: christophe.royonecea.fr

\section{O. Kepka}

Prague, Inst. Phys.

E-mail: oldrich.kepka@cern.ch

\begin{abstract}
We study the $W / Z$ pair production via two-photon exchange at the LHC and give the sensitivities on trilinear and quartic gauge anomalous couplings between photons and $W / Z$ bosons. Tagging the protons in the final state in the ATLAS Forward Physics detectors as an example allows to improve the reach on anomalous couplings by four orders of magnitude reaching the values predicted by extra-dimension theories.
\end{abstract}

International Conference on the Structure and the Interactions of the Photon including the 20th International Workshop on Photon-Photon Collisions and the International Workshop on High Energy Photon Linear Colliders

20 - 24 May 2013

Paris, France

${ }^{*}$ Speaker. 


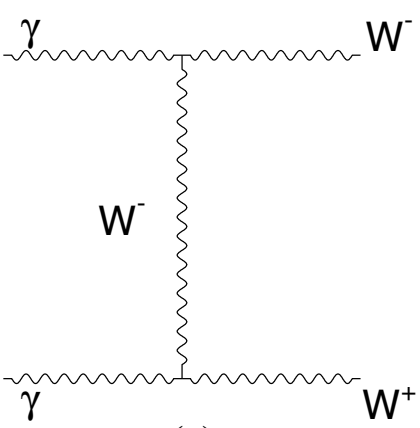

(a)

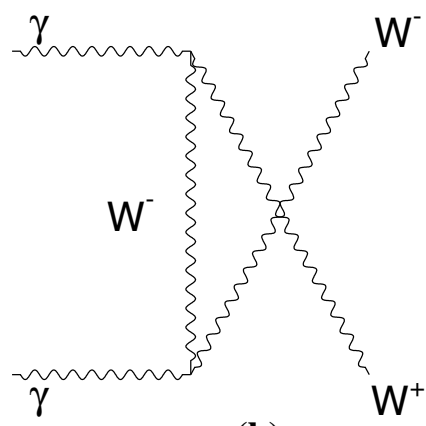

(b)

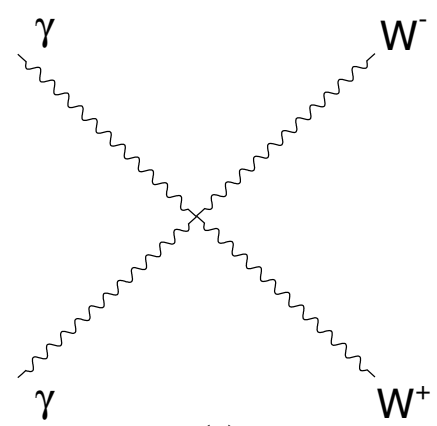

(c)

Figure 1: Diagrams contributing to $W$ boson pair production via photon exchange, with (a,b) triple $W W \gamma$ and (c) quartic $W W \gamma \gamma$ couplings.

\section{1. $W$ pair production via photon exchange}

Exclusive diffractive processes give a very clear experimental signature. These events are characterized by an object produced in the central detector, two intact protons diffracted at low angles from the beam, and no other products from the interaction (no energy loss or proton remnants).

Because of this unique experimental signature, forward proton detectors are needed to study exclusive diffractive processes in detail. One project implementing such detectors at the LHC is the ATLAS Forward Physics project (AFP), which is an upgrade of the ATLAS experiment. It consists of forward proton detectors to be installed on both sides of the ATLAS detector, about $220 \mathrm{~m}$ from the interaction point, in the LHC tunnel. The detectors will be installed in movable beam pipes, and include both silicon detectors (for tracking and proton momentum measurement) and timing detectors (to associate a detected proton to a primary vertex in the central detector, based on its time of flight). A similar project, the Precision Proton Spectrometers, has recently been approved by the CMS collaboration.

In this study [1], we focus on two-photon interactions at the LHC. More precisely, we study the QED process $p p \rightarrow p p W W$ (see Fig. 1): we effectively use the LHC as a photon collider to produce a pair of $W$ bosons. The cross section for this process is fairly large in the Standard Model $(\mathrm{SM}): \sigma_{p p \rightarrow p p W W}=95.6 \mathrm{fb}$ at $\sqrt{s}=14 \mathrm{TeV}$. Interestingly, a significant part of this cross section corresponds to high two-photon masses: $\sigma_{p p \rightarrow p p W W}\left(W=M_{X}>1 \mathrm{TeV}\right)=5.9 \mathrm{fb}$. This process has a high sensitivity to beyond standard model effects, especially anomalous gauge boson couplings (AQGCs).

We have used the photon equivalent approximation (Budnev flux [2]) in our computations. It is characterized by a low photon virtuality $\left(Q^{2}\right)$, but high photon energies are possible. In particular a significant part of the two-photon luminosity corresponds to high missing masses $M_{\gamma \gamma}=\sqrt{s \xi_{1} \xi_{2}}$ (where $\xi$ is the momentum fraction loss of the proton).

The parameterization of the AQGCs is based on Ref. [3], and only the lowest dimension operators that have the correct Lorentz invariant structure and fulfill $S U(2)_{C}$ custodial symmetry [4] are considered. Such operators involving two $W$ bosons and two photons final state are of dimension six: 


$$
\begin{aligned}
& \mathscr{L}_{6}^{0}=\frac{-e^{2}}{8} \frac{a_{0}^{W}}{\Lambda^{2}} F_{\mu \nu} F^{\mu v} W^{+\alpha} W_{\alpha}^{-}-\frac{e^{2}}{16 \cos ^{2} \Theta_{W}} \frac{a_{0}^{Z}}{\Lambda^{2}} F_{\mu \nu} F^{\mu v} Z^{\alpha} Z_{\alpha} \\
& \mathscr{L}_{6}^{C}=\frac{-e^{2}}{16} \frac{a_{C}^{W}}{\Lambda^{2}} F_{\mu \alpha} F^{\mu \beta}\left(W^{+\alpha} W_{\beta}^{-}+W^{-\alpha} W_{\beta}^{+}\right)-\frac{e^{2}}{16 \cos ^{2} \Theta_{W}} \frac{a_{C}^{Z}}{\Lambda^{2}} F_{\mu \alpha} F^{\mu \beta} Z^{\alpha} Z_{\beta}
\end{aligned}
$$

where $F^{\mu v}$ is the electromagnetic field strength tensor and $W_{\alpha}^{ \pm}$is the $W^{ \pm}$boson field. All anomalous parameters $a_{0}^{W}, a_{C}^{W}, a_{0}^{Z}$ and $a_{C}^{Z}$ are equal to zero in the SM. Here, only anomalous $\gamma \gamma W W$ and $\gamma \gamma Z Z$ couplings are considered, but many more are possible, for instance between photons or involving the Higgs boson.

$a_{0}$ and $a_{C}$ are the usual notation for the parametrized quartic coupling constants, where a nonzero $a_{0}$ could be due to an exchange of a heavy neutral scalar, while heavy charged fermions would contribute to both $a_{0}$ and $a_{C}$. The new scale $\Lambda$ is introduced so that the Lagrangian density has the correct dimension of four and is interpreted as the typical mass scale of new physics.

The $p p \rightarrow p p W^{+} W^{-}$cross section via photon exchange rises quickly at high energies when the anomalous coupling parameters are non-zero, and manifests itself in particular with the production of boosted $W$ boson pairs. In the SM, the $\gamma \gamma \rightarrow W W$ cross section is constant in the high-energy limit due to the cancellation between the relevant diagrams. When the new quartic terms are added, the cancellation does not hold and the cross section will grow to violate unitarity at high energies. This increase of the cross section can be regularized with a form factor that reduces the values of $a_{0}^{W}$ and $a_{C}^{W}$ at high energy while not modifying them at lower energies. Following a standard approach, we introduce the following form factor [5]:

$$
a_{i}^{W} \rightarrow \frac{a_{i}^{W}}{\left(1+M_{\gamma \gamma}^{2} / \Lambda_{\text {cutoff }}^{2}\right)^{2}},
$$

where $M_{\gamma \gamma}$ is the invariant mass of the two photons, and $\Lambda_{\text {cutoff }}$ is chosen to be either 0.5 or 1 $\mathrm{TeV}$, following the prescription of, e.g., Ref. [5]. In the following, we provide limits on anomalous couplings with and without form factors.

The best 95\% C.L. published limits on these anomalous parameters at the time of the communication came from the OPAL Collaboration from measurement of $W W \gamma, q \bar{q} \gamma \gamma$ and $v \bar{v} \gamma \gamma$ production [6] at the CERN LEP Collider:

$$
\begin{aligned}
-0.020<a_{0}^{W} / \Lambda^{2} & <0.020 \mathrm{GeV}^{-2} \\
-0.052 & <a_{C}^{W} / \Lambda^{2}<0.037 \mathrm{GeV}^{-2} \\
-0.007 & <a_{0}^{Z} / \Lambda^{2}<0.023 \mathrm{GeV}^{-2} \\
-0.029 & <a_{C}^{Z} / \Lambda^{2}<0.029 \mathrm{GeV}^{-2}
\end{aligned}
$$

These constraints were recently superseded by new results by new results from the D0 collaboration at the Tevatron [7], and from the CMS collaboration at the LHC Collider [8]:

$$
\begin{aligned}
& \left|a_{0}^{W} / \Lambda^{2}\right|<0.00015 \mathrm{GeV}^{-2} \quad\left(a_{C}^{W} / \Lambda^{2}=0, \Lambda_{\text {cutoff }}=500 \mathrm{GeV}\right) \\
& \left|a_{C}^{W} / \Lambda^{2}\right|<0.0005 \mathrm{GeV}^{-2} \quad\left(a_{0}^{W} / \Lambda^{2}=0, \Lambda_{\text {cutoff }}=500 \mathrm{GeV}\right)
\end{aligned}
$$


(a)

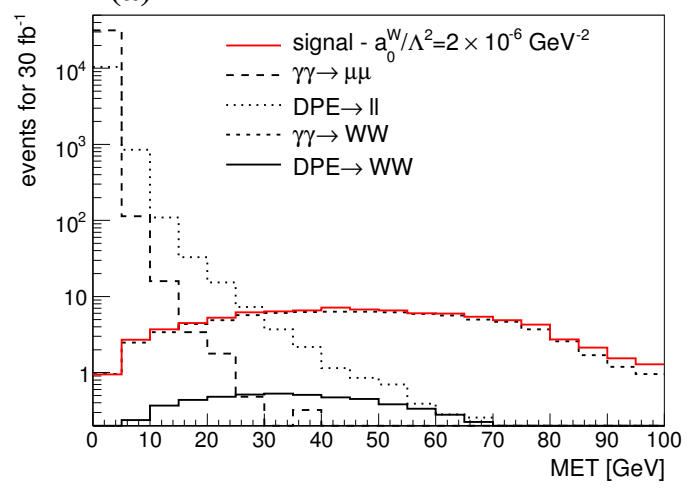

(b)

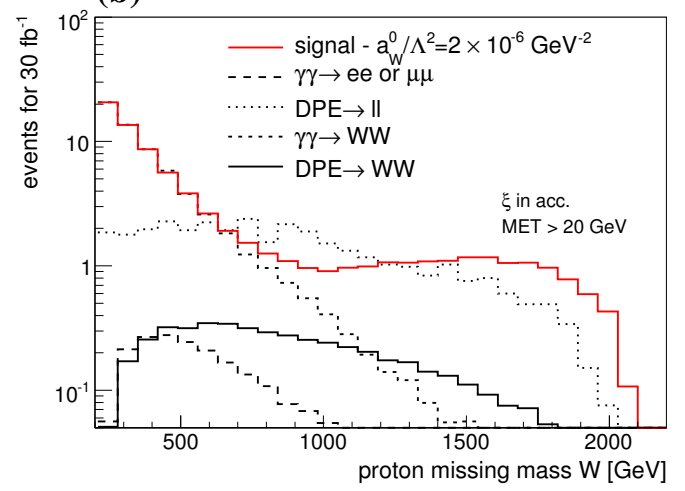

Figure 2: (a) Missing transverse energy distribution in the AFP detector acceptance cut and (b) proton missing mass in the AFP acceptance and after the cut on MET $>20 \mathrm{GeV}$ cut for signal and all backgrounds with $\mathscr{L}=30 \mathrm{fb}^{-1}$

\section{Fast simulation}

We are focusing on $W$ boson pairs produced through photon exchange between the incoming protons, with the $W$ bosons decaying leptonically. Experimentally, such events are characterized by two high momentum leptons in the central detector, two tagged protons in the forward detectors, and no other object associated to the primary vertex.

We assume that non-diffractive backgrounds, including inelastic $W W$ production, are perfectly rejected thanks to the detection of the intact protons. Thus the only backgrounds we are left with are dilepton production through photon exchange or double pomeron exchange (DPE) and $W W$ through DPE. However, dilepton production is characterized by back-to-back leptons and very small missing transverse energy (MET). Moreover, double pomeron exchange processes add the presence of energy flow in the forward regions and a higher number of tracks, because of the pomeron remnants.

In our study using fast simulation, background processes are rejected using kinematic cuts. At the preselection stage, we require two reconstructed leptons $(e e, e \mu$ or $\mu \mu)$ with $\left|\eta^{e, \mu}\right|<2.5$ and $p_{T}^{e, \mu}>10 \mathrm{GeV}$, two tagged protons (i.e. within the acceptance of the AFP detectors: $0.0015<\xi<$ 0.15 ), and nothing else associated to the primary vertex. Dilepton production is rejected thanks to a cut on missing transverse energy (MET $>20 \mathrm{GeV}$ ) and on the azimuthal opening angle between the two leptons $(\Delta \phi<3.13 \mathrm{rad})$. To reject DPE $W W$ production and increase the sensitivity to anomalous couplings, we cut on the mass $W$ of the central system $(W>800 \mathrm{GeV})$ and on the leading lepton $p_{T}\left(p_{T}^{\text {lep } 1}>160 \mathrm{GeV}\right)$.

The effect of these cuts is shown on Fig. 2 and in Tables 1 and 2. Despite a good efficiency on the signal, they allow to reject almost completely the background processes: less than 1 event is expected for $30 \mathrm{fb}^{-1}$.

The results of our study using a fast simulation of detector effects (with ATLFAST++ [9]) are shown in Table 3 and Fig. 3. The sensitivity on anomalous couplings that one could reach using our analysis techniques is up to four orders of magnitude better than that obtained at LEP. It is also one order of magnitude more sensitive than searches using $p p \rightarrow \ell^{ \pm} v \gamma \gamma$ [10]. 
events for $30 \mathrm{fb}^{-1}$ (fast simulation ATLFAST++)

\begin{tabular}{|c|cccc|}
\hline cut / process & $\gamma \gamma \rightarrow \ell \ell$ & $\gamma \gamma \rightarrow W W$ & $\mathrm{DPE} \rightarrow \ell \ell$ & $\mathrm{DPE} \rightarrow W W$ \\
\hline \hline$p_{T}^{\text {lep1,2}}>10 \mathrm{GeV}$ & 50619 & 99 & 18464 & 8.8 \\
$0.0015<\xi<0.15$ & 21058 & 89 & 11712 & 6.0 \\
$E_{T}>20 \mathrm{GeV}$ & 14.9 & 77 & 36 & 4.7 \\
$W>800 \mathrm{GeV}$ & 0.42 & 3.2 & 16 & 2.5 \\
$M_{l l} \notin[80,100]$ & 0.42 & 3.2 & 13 & 2.5 \\
$\Delta \phi<3.13 \mathrm{rad}$ & 0.10 & 3.2 & 12 & 2.5 \\
$p_{T}^{\text {lep } 1}>160 \mathrm{GeV}$ & 0 & 0.69 & 0.20 & 0.024 \\
\hline
\end{tabular}

Table 1: Rejection of the background by the successive application of the selection cuts. The number of events is normalized to $\mathscr{L}=30 \mathrm{fb}^{-1}$ of integrated luminosity. The lepton index lep corresponds to electrons or muons. The DPE $\rightarrow \ell \ell$ was generated with a minimum Drell-Yan mass $10 \mathrm{GeV}$.

events for $30 \mathrm{fb}^{-1}$ (fast simulation ATLFAST++)

\begin{tabular}{|c|cc|}
\hline cut / couplings (with f.f.) & $\mid a_{0}^{W} / \Lambda^{2}=5.4 \cdot 10^{-6} \mathrm{GeV}^{-2}$ & $\left|a_{C}^{W} / \Lambda^{2}\right|=20 \cdot 10^{-6} \mathrm{GeV}^{-2}$ \\
\hline \hline$p_{T}^{\text {lep1 }, 2}>10 \mathrm{GeV}$ & 202 & 200 \\
$0.0015<\xi<0.15$ & 116 & 119 \\
$E_{T}>20 \mathrm{GeV}$ & 104 & 107 \\
$W>800 \mathrm{GeV}$ & 24 & 23 \\
$M_{l l} \notin[80,100]$ & 24 & 23 \\
$\Delta \phi<3.13 \mathrm{rad}$ & 24 & 22 \\
$p_{T}^{l e p 1}>160 \mathrm{GeV}$ & 17 & 16 \\
\hline
\end{tabular}

Table 2: Selection of the signal by the successive application of the cuts. The number of events is given for integrated luminosity of $\mathscr{L}=30 \mathrm{fb}^{-1}$. The lepton index lep corresponds to electrons or muons.

\begin{tabular}{|c|c|c|c|}
\hline Couplings & OPAL limits & \multicolumn{3}{|c|}{$95 \%$ limits @ $\mathscr{L}=30(200) \mathrm{fb}^{-1}$} \\
& {$\left[\mathrm{GeV}^{-2}\right]$} & $\Lambda=\infty$ & $\Lambda=2 \mathrm{TeV}$ \\
\hline \multirow{2}{*}{$a_{0}^{W} / \Lambda^{2}$} & {$[-0.020,0.020]$} & $\begin{array}{c}1.2 \cdot 10^{-6} \\
\left(0.7 \cdot 10^{-6}\right)\end{array}$ & $\begin{array}{c}2.6 \cdot 10^{-6} \\
\left(1.4 \cdot 10^{-6}\right)\end{array}$ \\
\hline \multirow{2}{*}{$a_{0}^{Z} / \Lambda^{2}$} & {$[-0.052,0.037]$} & $\begin{array}{c}4.2 \cdot 10^{-6} \\
\left(2.4 \cdot 10^{-6}\right)\end{array}$ & $\begin{array}{c}9.4 \cdot 10^{-6} \\
\left(5.2 \cdot 10^{-6}\right)\end{array}$ \\
\hline \multirow{2}{*}{$a_{C}^{W} / \Lambda^{2}$} & {$[-0.007,0.023]$} & $\begin{array}{c}2.8 \cdot 10^{-6} \\
\left(1.1 \cdot 10^{-6}\right)\end{array}$ & $\begin{array}{c}6.4 \cdot 10^{-6} \\
\left(2.5 \cdot 10^{-6}\right)\end{array}$ \\
\hline \multirow{2}{*}{$a_{C}^{Z} / \Lambda^{2}$} & {$[-0.029,0.029]$} & $\begin{array}{c}1.0 \cdot 10^{-5} \\
\left(4.1 \cdot 10^{-6}\right)\end{array}$ & $\begin{array}{c}2.4 \cdot 10^{-5} \\
\left(9.2 \cdot 10^{-6}\right)\end{array}$ \\
\hline
\end{tabular}

Table 3: Expected 95\% confidence level intervals on the $W W \gamma \gamma$ and $Z Z \gamma \gamma$ anomalous quartic parameters using $\mathscr{L}=30 \mathrm{fb}^{-1}$ or $200 \mathrm{fb}^{-1}$ of data at high luminosity with forward detectors, and with or without the form factors applied. 


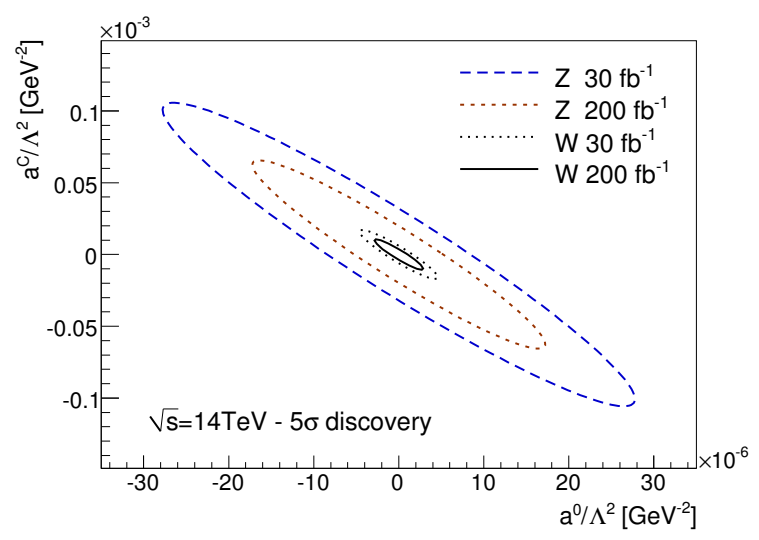

Figure 3: $5 \sigma$ discovery contours for all the $W W$ and $Z Z$ quartic couplings at $\sqrt{s}=14 \mathrm{TeV}$ for $\mathscr{L}=30 \mathrm{fb}^{-1}$ and $\mathscr{L}=200 \mathrm{fb}^{-1}$.

\section{Full simulation}

In the previous section, we were assuming that pile-up interactions and non-diffractive processes were perfectly rejected. The actual efficiency of exclusivity cuts has to be checked using full simulation, which is described in the present section. The exclusivity of the event is defined thanks to the proton time of flight (which allows to associate back the detected forward proton to a primary vertex in the interaction region), but also to tracks fitted to the primary vertex. Indeed, for signal, only two tracks are expected from the vertex: the two charged leptons from the decay of the $W$ bosons.

In this study with full simulation [11], in addition to the diffractive backgrounds mentioned earlier, we also considered single-diffractive $W W$ production, as well as non-diffractive backgrounds: top-antitop pair production, diboson, $W / Z+$ jets, and single top. We assume a 10 picosecond resolution for the proton timing detectors, and two scenarios with different instantaneous and integrated luminosities: $\mathscr{L}=40 \mathrm{fb}^{-1}$ and $\mu=23$ average interactions per bunch crossing, or $\mathscr{L}=200 \mathrm{fb}^{-1}$ and $\mu=46$.

The analysis procedure is kept the same as in our study using fast simulation. Table 4 confirms that our exclusivity cuts are able to completely get rid of the non-diffractive backgrounds. Once all the cuts are applied, we once again expect less than one background event, but a significant amount of signal from anomalous couplings.

The results obtained with the full simulation are presented in Table 5 and are similar to the ones presented earlier with fast simulation.

\section{Summary}

We have presented an interesting way of probing new physics in the electroweak sector of the SM, by studying $W$ exclusive pair production at the LHC. This study demonstrates that the LHC can also be seen as a photon collider, as it provides as it provides a good photon-photon luminosity. Rejection of inclusive backgrounds is crucial to this study, and in a high luminosity 
events for $300 \mathrm{fb}^{-1}$ and $\mu=46$ (full simulation)

\begin{tabular}{|l|c|c|c|c|c|c|}
\hline Cuts & Top & Dibosons & Drell-Yan & W/Z+jet & Diffr. & $a_{0}^{W} / \Lambda^{2}=5 \cdot 10^{-6} \mathrm{GeV}^{-2}$ \\
\hline \hline $\begin{array}{l}\text { timing }<10 \mathrm{ps} \\
p_{T}^{\text {lep1 }}>150 \mathrm{GeV} \\
p_{T}^{\text {lep2 }}>20 \mathrm{GeV}\end{array}$ & 5198 & 601 & 20093 & 1820 & 190 & 282 \\
\hline$M_{l l}>300 \mathrm{GeV}$ & 1650 & 176 & 2512 & 7.7 & 176 & 248 \\
\hline nTracks $\leq 3$ & 2.8 & 2.1 & 78 & 0 & 51 & 71 \\
\hline$\Delta \phi<3.1$ & 2.5 & 1.7 & 29 & 0 & 2.5 & 56 \\
\hline$m_{X}>800 \mathrm{GeV}$ & 0.6 & 0.4 & 7.3 & 0 & 1.1 & 50 \\
\hline$p_{T}^{\text {lep1 }}>300 \mathrm{GeV}$ & 0 & 0.2 & 0 & 0 & 0.2 & 35 \\
\hline
\end{tabular}

Table 4: Number of expected signal and background events for $300 \mathrm{fb}^{-1}$ at pile-up $\mu=46$. A time resolution of $10 \mathrm{ps}$ has been assumed for background rejection. The diffractive background comprises production of QED diboson, QED dilepton, diffractive $W W$, double pomeron exchange $W W$.

\begin{tabular}{|c|c|c|}
\hline & \multicolumn{2}{|c|}{$a_{0}^{W} / \Lambda^{2}$ Sensitivity } \\
& $5 \sigma$ & $95 \%$ C.L. \\
\hline \hline $\mathscr{L}=40 \mathrm{fb}^{-1}, \mu=23$ & $5.5 \cdot 10^{-6}$ & $2.4 \cdot 10^{-6}$ \\
$\mathscr{L}=300 \mathrm{fb}^{-1}, \mu=46$ & $3.2 \cdot 10^{-6}$ & $1.3 \cdot 10^{-6}$ \\
\hline
\end{tabular}

Table 5: Reach on anomalous couplings obtained in $\gamma$-induced processes after tagging the protons in the final state in the AFP detectors. The $5 \sigma$ discovery and $95 \%$ C.L. limits are given for an integrated luminosity of $40 \mathrm{fb}^{-1}$ at $\mu=23$ and $300 \mathrm{fb}^{-1}$ at $\mu=46$ as obtained using the full MC and the background processes described in the text.

environment it requires forward proton detectors with excellent timing resolution (e.g. AFP) to be installed. We have proposed a way to improve the current limits on anomalous quartic gauge boson couplings between the photon and the $W$ boson, by up to four orders of magnitude compared to the LEP limits. A similar study using diphoton events [12] has proved to provide good sensitivity on anomalous quartic photon couplings.

\section{References}

[1] E. Chapon, C. Royon and O. Kepka, Anomalous quartic $W W \gamma \gamma, Z Z \gamma \gamma$, and trilinear $W W \gamma$ couplings in two-photon processes at high luminosity at the LHC, Phys. Rev. D 81 (2010) 074003 [arXiv:0912.5161 [hep-ph]].

[2] V. M. Budnev, I. F. Ginzburg, G. V. Meledin and V. G. Serbo, The Two photon particle production mechanism. Physical problems. Applications. Equivalent photon approximation, Phys. Rept. 15 (1975) 181.

[3] G. Belanger and F. Boudjema, $\gamma \gamma \rightarrow W^{+} W^{-}$and $\gamma \gamma \rightarrow Z Z$ as tests of novel quartic couplings, Phys. Lett. $B 288$ (1992) 210. In the present study, the $a_{0}^{Z}$ and $a_{C}^{Z}$ parameters are assumed to be zero.

[4] R. A. Diaz and R. Martinez, The Custodial symmetry, Rev. Mex. Fis. 47, 489 (2001) [hep-ph/0302058]. 
[5] O. J. P. Eboli, M. C. Gonzalez-Garcia, S. M. Lietti and S. F. Novaes, Anomalous quartic gauge boson couplings at hadron colliders, Phys. Rev. D 63, 075008 (2001) [hep-ph/ 0009262$].$

[6] G. Abbiendi et al. [OPAL Collaboration], Constraints on anomalous quartic gauge boson couplings from $v \bar{v} \gamma \gamma$ and $q \bar{q} \gamma \gamma$ events at LEP-2, Phys. Rev. D 70, 032005 (2004) [hep-ex/ 0402021 ].

[7] V. M. Abazov et al. [D0 Collaboration], Search for anomalous quartic WW $\gamma \gamma$ couplings in dielectron and missing energy final states in p $\bar{p}$ collisions at $\sqrt{s}=1.96 \mathrm{TeV}$, Phys. Rev. D 88 (2013) 012005 [arXiv:1305.1258 [hep-ex]].

[8] S. Chatrchyan et al. [CMS Collaboration], Study of exclusive two-photon production of $W^{+} W^{-}$in $p p$ collisions at $\sqrt{s}=7 \mathrm{TeV}$ and constraints on anomalous quartic gauge couplings, JHEP 1307 (2013) 116 [arXiv:1305.5596 [hep-ex]].

[9] ATLFast++ package for ROOT, http://root.cern.ch/root/Atlfast.html.

[10] P. J. Bell, Quartic Gauge Couplings and the Radiation Zero in $p p \rightarrow \ell^{ \pm} v \gamma \gamma$ events at the LHC, Eur. Phys. J. C 64 (2009) 25 [arXiv:0907.5299 [hep-ph] ].

[11] Letter of Intent for the Phase-I Upgrade of the ATLAS Experiment, CERN-LHCC-2011-012 / LHCC-I-020 (2011).

[12] S. Fichet, G. von Gersdorff, O. Kepka, B. Lenzi, C. Royon and M. Saimpert, Probing new physics in diphoton production with proton tagging at the Large Hadron Collider, [arXiv: 1312.5153 [hep-ph]]. 